This is the postprint version of the following article: Severoni, E; Maniappan, S; Liz-Marzán, LM; Kumar, J; García de Abajo, JF; Galantini, L Plasmon-Enhanced Optical Chirality through Hotspot Formation in Surfactant-Directed Self-Assembly of Gold Nanorods. ACS Nano DOI: $10.1021 /$ acsnano.0c03997

This article may be used for non-commercial purposes in accordance with ACS Terms and Conditions for Self-Archiving. 


\section{Plasmon-Enhanced Optical Chirality through Hotspot Formation in Surfactant-Directed Self- Assembly of Gold Nanorods}

Emilia Severoni $^{\dagger * *}$, Sonia Maniappan ${ }^{\S * *}$, Luis M. Liz-Marzán ${ }^{\ddagger}$, Jatish Kumar*§ ${ }^{*}$ F. Javier García de Abajo* ${ }^{+}$, Luciano Galantini* ${ }^{\dagger}$

†Dipartimento di Chimica, “Sapienza” Università di Roma, P. le A. Moro 5, 00185 Roma, Italy

$\S^{\S}$ Department of Chemistry, Indian Institute of Science Education and Research (IISER) Tirupati, Tirupati 517 507, India

${ }^{\ddagger}$ CIC biomaGUNE, Basque Research and Technology Alliance (BRTA), Paseo de Miramón 182, 20014 Donostia-San Sebastián, Spain

"Ikerbasque, Basque Foundation for Science, 48013 Bilbao, Spain

${ }^{\infty}$ Centro de Investigación en Red de Bioingeniería, Biomateriales y Nanomedicina (CIBER-BBN),

Paseo de Miramón 182, 20014 Donostia-San Sebastián, Spain

"ICFO-Institut de Ciencies Fotoniques, The Barcelona Institute of Science and Technology, 08860 Castelldefels (Barcelona), Spain

${ }^{+}$ICREA-Institució Catalana de Recerca i Estudis Avançats, Passeig Lluís Companys 23, 08010 Barcelona, Spain

**These authors contributed equally to the work.

Corresponding authors’ e-mail: luciano.galantini@uniroma1.it (LG);

javier.garciadeabajo@nanophotonics.es (FJGA); jatish@iisertirupati.ac.in (JK) 
ABSTRACT. Plasmonically enhanced optical dichroism has attracted substantial interest for its application in optical sensing, where the interplay between chirality emanating from both molecules and plasmon-supporting structures has been regarded as a critical ingredient. Here we experimentally demonstrate that suitably self-assembled achiral plasmonic nanostructures produce a high degree of enhancement in the optical dichroism observed from chiral molecules placed in their vicinity. Specifically, we identify near-field enhancement associated with plasmonic hotpots as the mechanism enabling our observation of visible-NIR circular dichroism emanating from small amounts of chiral molecules. Our structures consist of linear arrays of gold nanorods obtained by introducing chiral anionic surfactants, such as modified bile salts, which lead to selective destabilization of a cetyltrimethylammonium bromide coating layer on Au nanorods, thereby promoting tip-to-tip oriented assembly. The proposed mechanism of plasmonicallyenhanced circular dichroism is supported by deriving a simple, yet general theoretical formalism that confirms the observed results, revealing the role of optical hotspots at the gaps of linear tipto-tip nanorod assemblies as the origin of enhancement in the dichroism from chiral molecules. Importantly, it is the refractive rather than the absorption-mediated chiral response of the molecules what produces dichroism in the vis-NIR plasmonic regime, far from their UV absorption resonances. The observed self-assembly mechanism suggests that chiral analytes not directly interacting with the nanorod surfaces, but just able to destabilize their caps, can be revealed by a CD signature in the plasmonic region, thereby supporting potential applications in ultrasensitive analysis.

KEYWORDS. gold nanorods, plasmonic circular dichroism, bile salts, chiral plasmons, catanionic mixtures, plasmonic hotspot 
The modulation of physical properties in metal nanoparticles through their controlled organization has attracted significant attention in the context of nanoscience and nanotechnology. ${ }^{1-3}$ When assembled at close distances, localized surface-plasmon resonances (LSPR) at individual nanoparticles can couple, resulting in the generation of hybridized plasmons that can generate hotspots with enhanced optical electric field at nanoparticle junctions. ${ }^{4}$ Such hotspots have been effectively used for the enhancement of spectroscopic signals in techniques like surface-enhanced Raman scattering (SERS) ${ }^{5}$ and metal-enhanced fluorescence (MEF). ${ }^{6}$ In contrast to linear arrangements, the helical organization of metal nanoparticles has been shown to lead to interesting chiroptical properties, thereby facilitating the design of materials that may find applications in the areas of optics, biosensing and catalysis, among others. ${ }^{7-9}$ Different strategies adopted for the generation of plasmonic chirality include: (i) synthesis of inherently chiral particles; ${ }^{9-11}$ (ii) chiral assemblies of nanoparticles driven by various helical templates such as peptides, ${ }^{12}$ proteins, ${ }^{13}$ amino acids, ${ }^{14}$ DNA, ${ }^{15}$ and cellulose nanocrystals; ${ }^{16}$ and (iii) hotspot-driven plasmonic chirality. ${ }^{17,18}$ Chiral induction in the first two cases is due to the symmetry breaking imposed by geometrical factors in the arrangement or internal structure of the particles, whereas hotspot-driven optical activity is the result of enhancement of the chiral signal from molecules exposed to nanoparticle-mediated plasmon-enhanced electric fields (i.e., the molecular chiral signal is amplified by interaction with the particle plasmons, but the latter do not exhibit intrinsic chirality). While comprehensive studies have been conducted on the experimental and theoretical understanding of chirality induced by geometrical factors, including the prediction of opticalcavity-mediated transfer of chirality from a chiral molecule to the Raman response of an achiral molecule, ${ }^{19}$ the investigation on hotspot-enhanced plasmonic chirality has been scarce and still requires both careful experimental design and a specific theoretical framework. 
In this article, we demonstrate the generation of plasmonically-enhanced circular dichroism (CD) through exposing chiral molecules to optical hotspots created in Au nanorod (AuNR) assemblies produced by directed tip-to-tip linking. Whereas visible-NIR dichroism from the molecules in dispersion is hardly measurable, the effect of near-field enhancement due to plasmonic hotspots in the AuNR assemblies results in a noticeable CD signal. Rather than using conventional linker molecules, we adopt an approach in which chiral co-surfactants are employed to selectively deprotect the tips of nanorods, thereby promoting selective tip-to-tip self-assembly of AuNRs, induced by a purposefully designed anionic surfactant (Figure 1a) derived from bile salts (BSs). We further show that assembled AuNRs display a larger number of hotspots than those assembled through addition of a conventional head-tail surfactant (sodium dodecylsulfate (SDS), Figure 1b, and natural BSs, Figure 1c), thus emerging as suitable structures for the investigated mechanism of CD enhancement. The increase in chirality signal at the plasmonic wavelength range is due to the presence of AuNRs and the resulting enhanced electric field prevailing at the linear junctions between AuNR tips. We further introduce a simple, general theoretical formalism based on straightforward manipulation of near-field simulations that supports the mechanism of plasmonmediated molecule-light interaction to interpret our observation of enhanced optical chirality. 

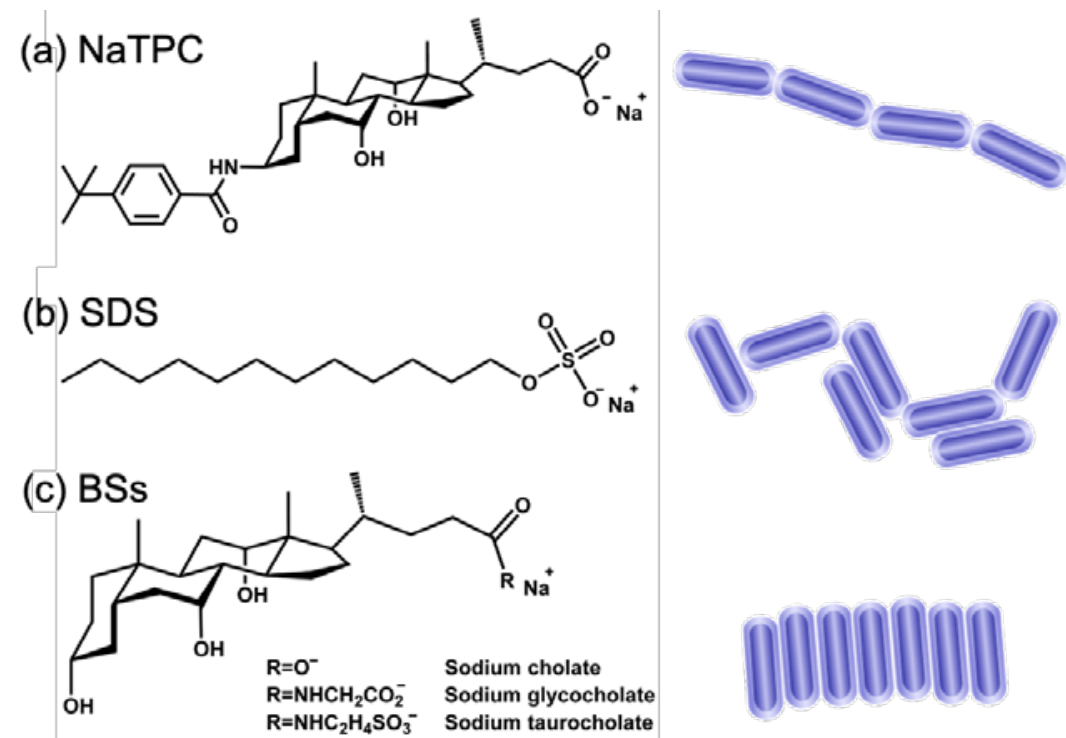

Figure1. Molecular structures and the corresponding self-assembly patterns induced by particle linkers used in this study: (a) sodium p-tertbutyl phenyl amido substituted cholate (NaTPC); (b) sodium dodecyl sulphate (SDS); and (c) natural bile salts (BSs).

\section{RESULTS AND DISCUSSION}

BSs are a class of steroidal biological surfactants, comprising a characteristic four-ring system of steroids and a lateral chain ending with a charged head-group. ${ }^{20-22}$ Hydroxyl groups are located on the condensed four-ring system, and in some BS families these are oriented on one of the two sides of the steroidal skeleton, thereby providing facial amphiphilicity to the molecule. In certain cases, the amphiphilic structure has been modified to synthesize derivatives with significant potential in pharmacological applications ${ }^{23,24}$ and nanomedicine. ${ }^{23}$ Among these derivatives, the $p$-tertbutyl phenyl amido-substituted cholate (NaTPC) used in this work (Figure 1a) features a bulky hydrophobic substituent, far away from the charged head-group. This peculiar feature in NaTPC enhances its hydrophobicity and breaks the facial amphiphilicity. ${ }^{25-27}$ We observed that the addition of NaTPC to cetyltrimethylammonium bromide (CTAB)-capped AuNRs triggers selective 
tip-to-tip self-assembly. We suggest that the supramolecular packing of NaTPC within the CTAB layer on the nanorod surface, as a consequence of inclusion of the oppositely charged amphiphile, is responsible for such AuNR aggregation.

CTAB-protected AuNRs with an aspect ratio of 4.0 (average length of $26 \pm 4 \mathrm{~nm}$ and average width of $6.5 \pm 2 \mathrm{~nm}$ ), ${ }^{28}$ and a longitudinal LSPR band centered around $815 \mathrm{~nm}$, were selected for initial investigations. Addition of a low concentration of NaTPC $(20 \mu \mathrm{M})$ to AuNRs (1.2 nM) in water did not show any significant effect on the extinction spectrum. However, when NaTPC concentration was increased up to a critical value $(25 \mu \mathrm{M})$, a gradual decrease in the intensity of the longitudinal LSPR band of AuNRs was observed, with the concomitant emergence of a redshifted band in the NIR region (Figure 2a). The extinction spectra exhibited a time-dependent shift, emerging band reaching ca. $980 \mathrm{~nm}$ after a time period of $20 \mathrm{~min}$. No changes were found to occur in the transverse LSPR band. Studies of the concentration dependence revealed dramatic changes in the intensities of the longitudinal LSPR and the coupled-plasmon bands at the critical concentration of the cosurfactant (Figure S1). Based on earlier reports, the observed extinction spectral changes can be attributed to the preferential tip-to-tip alignment of AuNRs. ${ }^{2,3}$ Moreover, the concomitant broadening of the longitudinal LSPR peak supports the formation of AuNR assemblies with varying orientations and chain lengths. Investigation of the chiral properties was carried out by means of circular dichroism (CD) measurements. CD spectra remained silent for AuNR dispersions in water, both before and after addition of a small amount of co-surfactant, which suggests the absence of chirality enhancement of NaTPC in the presence of individual (noninteracting) AuNRs in dispersion. Interestingly, a well-defined CD signal was observed when marked spectral changes were noticed in the extinction bands, which indicates the induction of 
plasmon-mediated chirality in the resulting AuNR assemblies (Figure 2b). CD spectra recorded over the whole accessible wavelength range at the NaTPC concentration used to induce the tip-totip assembly of AuNRs revealed effects on the optical activity of the co-surfactant (Figure S2). Free NaTPC in solution exhibited a very weak positive CD signal in the UV range, at a wavelength corresponding to the absorption maximum. Similar experiments in the presence of AuNRs resulted in $\mathrm{CD}$ with a more intense negative signal in the same region. The observed effect on molecular CD signals is in agreement with those found for other chiral molecules interacting with nanoparticles ${ }^{29}$ or entrapped at hotspots ${ }^{30}$ of similar nanoparticle assemblies.
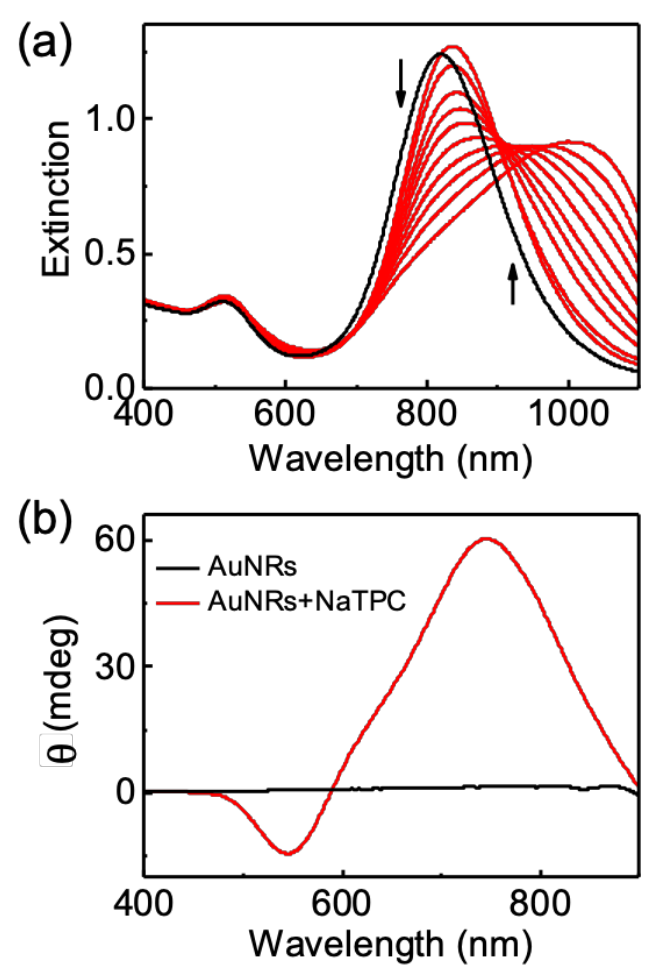

Figure 2. (a) Extinction spectra of AuNRs (1.2 nM) in water (black curve) and spectral changes upon addition of NaTPC $(25 \mu \mathrm{M})$ to a colloidal dispersion of AuNRs in water (red curves). (b) The corresponding CD spectra of AuNRs in water (black curve) and 20 min after the addition of NaTPC (red curve). 
To obtain further insight into the origin of CD signals in the plasmonic region, experiments were carried out using two sets of AuNRs possessing longitudinal LSPR bands at $695 \mathrm{~nm}$ and $820 \mathrm{~nm}$ (Figure S3), respectively. Bisignate CD signals in the spectral region of the corresponding coupled longitudinal plasmon band were observed in both cases. The presence of a first negative peak at higher wavelength is clear in NRs with a longitudinal LSPR at $695 \mathrm{~nm}$. However, CD features are missing for AuNRs with their longitudinal LSPR band at $820 \mathrm{~nm}$ within the detection limit of the instrument. The strong bisignate Cotton effect with a zero-crossover coinciding with the extinction maximum confirms the role of longitudinal hybridized plasmons in the generation of CD signals. The weaker CD signal present at a lower wavelength can be attributed to the effect of transverse plasmons. Similar modes were previously reported in AuNR assemblies linked by cysteine molecules. ${ }^{30}$ An anisotropy factor of $8 \times 10^{-3}$ was estimated by considering the maximum of the CD curve corresponding to the hybrid-plasmons band (see calculations in SI). A plot of CD intensity as a function of AuNR concentration clearly established the existence of a critical concentration for AuNR aggregation to take place (Figure S4). No appreciable CD signals were observed during the initial stages (below such a critical concentration), indicating that plasmon-enhanced chirality is not exhibited by individual AuNRs, but only by AuNR assemblies. Additionally, an increase in co-surfactant concentration well above the critical value did not enhance the CD signals further, indicating a saturation of the aggregation process. This saturation behavior is consistent with enhancement of chirality associated with the junctions between nanorods (see below), the number of which quickly approaches an asymptotic value (one hotspot per AuNR) when the average size of the ensembles exceeds a few AuNRs. 
Dynamic light scattering (DLS) measurements performed on an AuNR dispersion titrated with NaTPC showed that the hydrodynamic diameter remained unchanged at low NaTPC concentrations, whereas an abrupt increase was registered at the critical concentration of the steroidal surfactant (Figure S5). These results confirm that a threshold NaTPC concentration is required to trigger the aggregation of AuNRs. Starting from an electrophoretic mobility value of $+2.4 \pm 0.3 \mu \mathrm{m} \cdot \mathrm{cm} / \mathrm{Vs}$ for CTAB-capped AuNRs, a progressive decrease was recorded upon addition of NaTPC, until a leap in hydrodynamic diameter was reached. Interestingly, a still positive value of $+0.499 \pm 0.006 \mu \mathrm{m} \cdot \mathrm{cm} / \mathrm{Vs}$ was measured for the electrophoretic mobility at the assembly-triggering surfactant concentration, meaning that overcompensation was not reached.

Further insight into the nature of AuNR-NaTPC interactions was obtained from transmission electron microscopy (TEM) images. No specific patterns were observed for the AuNR dispersion drop-casted after addition of a low concentration $(10 \mu \mathrm{M})$ of NaTPC. However, at the critical concentration, TEM images showed preferential tip-to-tip assembly of AuNRs, in agreement with the changes observed in the measured extinction spectra. Smaller chains were observed at the initial stages, and continued extending in length over time. Long AuNR chains with preferential tip-to-tip assembly were observed after a period of $20 \mathrm{~min}$ (Figure 3c). Statistical analysis revealed 84\% of tip-to-tip contacts in the TEM images (Figure S6). 


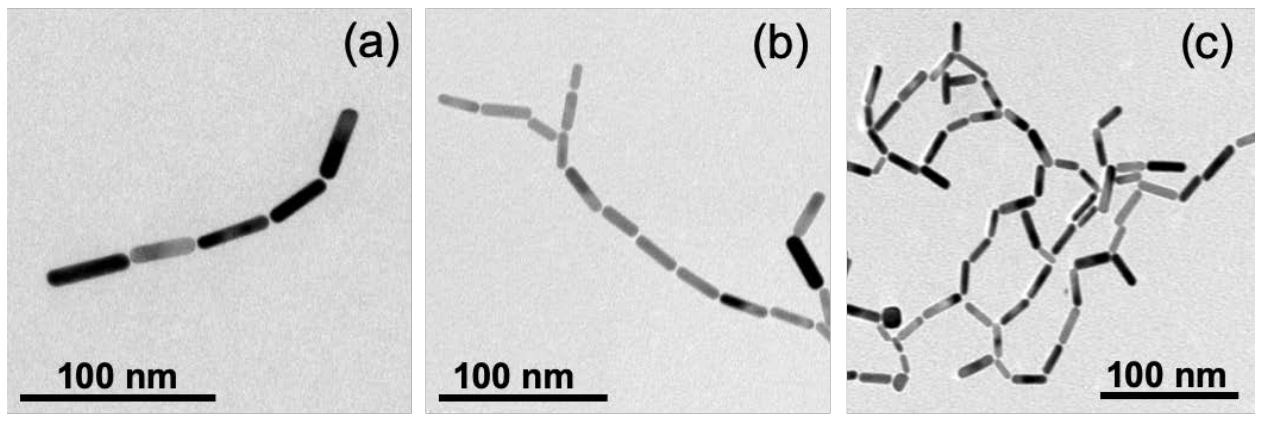

Figure 3. TEM images showing self-assembly of AuNRs at (a) $5 \mathrm{~min}$, (b) $10 \mathrm{~min}$, and (c) $20 \mathrm{~min}$ after addition of $25 \mu \mathrm{M}$ NaTPC to a colloidal dispersion of AuNRs in water.

A comparative analysis demonstrated that a conventional surfactant, such as SDS, can also induce AuNR assembly at relatively higher concentrations. However, a much lower selectivity was observed in this case, as only $45 \%$ of tip-to-tip contacts were counted from SEM images of SDSinduced AuNR aggregates (Figures S7c,d and S6). UV-vis and DLS measurements indicated a progressive aggregation of AuNRs when titrated with SDS, as opposed to the abrupt spectral and particle-size changes at the critical concentration, observed when using NaTPC (Figure S7a,b). Control experiments were also carried out using natural bile salts, namely, sodium cholate, glycocholate, and taurocholate (Figures 1c, S8). In all cases, addition to AuNR dispersions induced a gradual blue shift in the longitudinal LSPR and a redshift in the transverse LSPR bands, pointing toward side-by-side AuNR assembly (Figure S8). ${ }^{14}$ A BS concentration of ca. $260 \mu \mathrm{M}$ was required for aggregation, which is much larger than the co-surfactant concentration needed to trigger self-assembly in the AuNR-NaTPC system. CD signals of significantly lower intensity than those exhibited by AuNR-NaTPC mixtures were recorded in this case.

Basic principles for the self-assembly of conventional surfactants suggest that negatively charged amphiphiles are likely to infiltrate the CTAB bilayer on the nanorod surface, with the hydrophobic 
chain toward the interior and the charged head-group facing the external surface. ${ }^{31-34}$ The electrostatic attraction between positively charged head-groups of CTAB and the negatively charged anionic amphiphiles favor a closer packing of the head-groups, leading to an increase of the average packing parameter of the mixed bilayer. Due to the increase in the packing parameter, anionic and cationic surfactants (forming micelles in pure solutions) assemble into aggregates with a lower curvature when mixed. In surfactant-capped nanoparticles, the curvature is however imposed by the shape of the nanoparticles. Nanorods, for example, exhibit lateral facets with quasicylindrical curvature and tips with quasi-spherical curvature. According to the same principles of surfactant self-assembly, we thus conclude that anionic surfactants are more easily internalized in the capping bilayer at the lateral sides of nanorods because of the lower curvature of the bilayer. With this background knowledge, we anticipate a different scenario when an anionic surfactant is progressively added to the AuNR dispersion: (i) it starts with internalization of co-surfactant, leading to both partial charge neutralization and stabilization of the bilayer, up to a limiting fraction of internalized co-surfactant; (ii) at a concentration well above the limiting fraction, the bilayer is destabilized; (iii) the limiting fraction is lower for higher curvatures (i.e. destabilization occurs at lower concentrations at the AuNR tips); and (iv) both charge neutralization and destabilization of the bilayer can drive nanorod assembly.

On the basis of this general framework, the results obtained for the different surfactant additives can be explained as summarized in Scheme 1. Internalization in the bilayer is largely favored for SDS, which features a tail that perfectly fits the packing between $\mathrm{CTA}^{+}$chains in the bilayer interior. Accordingly, SDS is internalized at both the ends and the lateral facets of AuNRs. Owing to the higher concentration of the co-surfactant used, the SDS:CTA ${ }^{+}$ratio remains high in this 
case. When the limiting stabilizing fraction is reached at the end caps, the lateral surfaces are also neutralized (Scheme 1a). As a result, tip-to-tip assembly (due to destabilization at caps) is not selective in this case, and side-by-side assembly (due to charge neutralization) is equally favored. However, for NaTPC, which has an intermediate amphiphilic structure between the head-tail feature of SDS and the facial amphiphilicity of natural BSs, bilayer infiltration is less favorable than for SDS. This is because the steroidal skeleton of condensed rings can badly accommodate in between $\mathrm{CTA}^{+}$chains. Hence, destabilization of the bilayer occurs at a significantly lower fraction. This means that, when the caps at the tips are destabilized, the fraction of co-surfactant at the lateral surface is insufficient for charge neutralization. As a consequence, the lateral bilayer carries a high positive charge, leading to selective tip-to-tip assembly (Scheme 1b). The resulting AuNR junctions can act as hotspots in which the trapped chiral molecules experience an enhanced optical electric field, thereby inducing chiroptical activity at the wavelength of the coupled plasmon modes.

Unlike the NaTPC derivative, natural BSs cannot easily intercalate the CTAB bilayer, as they possess a well-defined facial amphiphilicity and a short molecular length compared to $\mathrm{CTA}^{+}$. However, they can induce nanoparticle assembly by a different mechanism (Scheme 1c). Indeed, the added surfactant molecules interact electrostatically through their carboxylate groups, with the $\mathrm{CTA}^{+}$head groups and the association between the hydrophobic faces of electrostatically bound BSs of different nanorods acting as linkers for nanoparticle assembly. Due to the larger surface area and high number of exposed positive charges on the lateral surface, side-by-side is preferred over tip-to-tip assembly. The chiral nature of BSs justifies the observed chirality enhancement triggered by coupling to the surface-plasmon modes of AuNRs. 
(a)

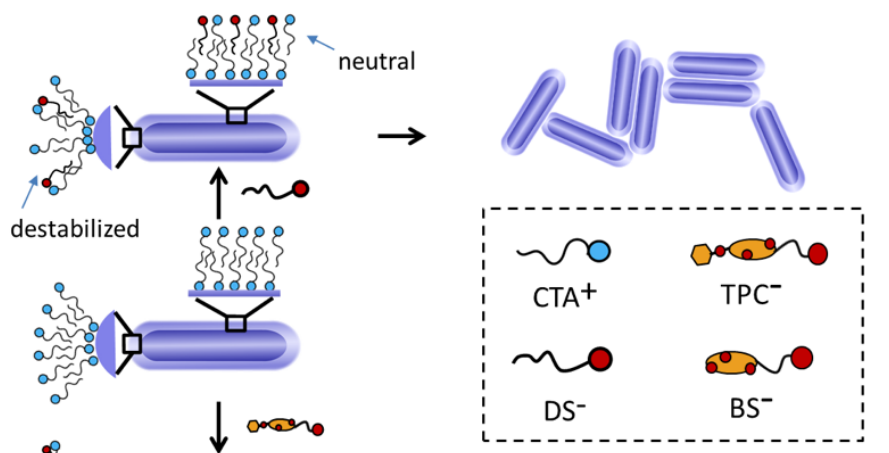

(b)

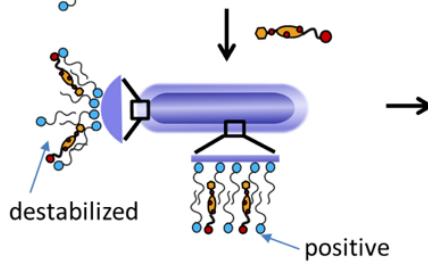

(c)
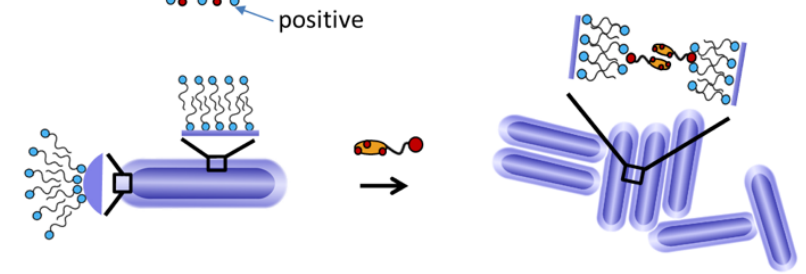

Scheme 1. Scheme illustrating the assembly of AuNRs induced by (a) SDS, (b) NaTPC, and (c) natural bile salts.

Previous reports demonstrate that, even if strong CD features of chiral molecules are typically emerging in the UV spectral range, far from the visible region (i.e., from the region in which nanoparticle plasmons occur), plasmon excitation can strongly interact with dipoles of chiral molecules and generate a CD response. ${ }^{17,18,30,35}$ The dominant term in the CD signal arises from the interference of the incident field and the field induced by a chiral molecular dipole inside the nanoparticle, and is proportional the optical rotatory dispersion of the molecule. ${ }^{36}$ The effect is amplified due to the enhancement of the electric fields at plasmonic hotspots. We measured specific optical rotations (i.e., in molecules without plasmonic nanoparticles) at a wavelength of $589.3 \mathrm{~nm}$ (i.e., $[\alpha]_{\mathrm{D}}$ in units of $\mathrm{deg} \cdot \mathrm{mL} \cdot \mathrm{g}^{-1} \cdot \mathrm{dm}^{-1}$ ) of $40 \pm 8$ for NaTPC and $23 \pm 5$ for the BS precursor sodium cholate. These data, along with those reported in the literature for natural BSs, ${ }^{37}$ 
demonstrate that the optical rotatory dispersion of the chiral co-surfactants used in our experiments is relevant in the plasmonic region, thereby supporting a mechanism based on the enhancement of intrinsic (but weak) molecular chirality at the plasmonic hotspots of AuNRs. Despite these evidences, we cannot completely rule out a different explanation for the observed CD in the plasmonic region, namely chirality emerging from a helical arrangement of the assembled nanorods, driven by the chiral co-surfactants at tip-to-tip junctions. We provide below a theoretical description of how intrinsic molecular $\mathrm{CD}$ can be enhanced by plasmons at nanoparticle assemblies.

Earlier theoretical models on hotspot-induced plasmonic chirality were based on the Coulombic interaction between an achiral NP dimer and the molecular dipole at the dimer junction. . $^{17,18,36}$ Plasmonically-induced CD was calculated for different NP sizes and gap separations, in which the chiral molecules were modelled as molecular dipoles having CD features in the UV region. Plasmon-enhanced CD features arise due to an interference between external and induced fields inside a NP-molecule hybrid complex. In order to obtain further insight into this concept and explore its role in the present study, we introduce a theoretical formalism that allows us to relate the observed chirality to a CD local enhancement (CDLE), and apply it for the particular case of tip-to-tip assembly of AuNRs using chiral co-surfactants. In contrast to previous studies, which were restricted to dimers, we extend our CDLE calculations to linear arrays containing several AuNRs. More interestingly, we obtain CD field enhancement plots as a function of the position of chiral molecules, which are useful to understand the contribution to the observed CD signal coming from different positions in the neighborhood of the NPs. This theoretical framework can further assist the design of nanostructures and assemblies with enhanced chiro-optical properties. 
We used the boundary-element method $(\mathrm{BEM})^{38,39}$ to calculate the electromagnetic near-field distribution and the far-field extinction produced by externally-illuminated AuNR linear arrays. In particular, we simulated the spectral dependence of the extinction cross section for an individual AuNR (26 nm end-to-end length, aspect ratio 4) and arrays with increasing AuNR numbers. The spectrum for an individual AuNR showed a dominant feature associated with its longitudinal plasmon at $~ 800 \mathrm{~nm}$, accompanied by a transverse plasmon mode at a shorter wavelength. As more AuNRs are brought together (linear arrays with $2 \mathrm{~nm}$ tip-to-tip separation), the initial modes are hybridized, giving rise to redshifted plasmonic features (Figure S9). Interestingly, the extinction cross section takes similar absolute values when normalized per nanorod in the structure, revealing that it is the bulk of the particles and not the gap regions where absorption is dominant (i.e., the existence of plasmonic hotspots does not seem to affect the overall efficiency of the structures to absorb light, relative to dispersed NPs). In other words, optical absorption, which originates in ohmic losses inside the metal, is proportional to the field intensity inside it, which the near-field maps in Figure S9 reveal to be maximum close to the center of the AuNRs. In contrast, the overall near field takes maximum values in the gap regions immediately outside the metal. ${ }^{38}$

The calculated extinction spectra are in reasonable agreement with the effects observed experimentally: for individual AuNRs the experimental spectrum is broadened due to the finite size distribution of the particles. As AuNRs aggregate into mainly linear arrays (small angles between AuNRs have been shown to have a negligible effect in the spectrum), ${ }^{40}$ we also expect a wide distribution of cluster sizes, giving rise to an overall redshift in the longitudinal plasmon band and substantial spectral broadening. In the current work, we are interested in the field enhancement 
acting on molecules deposited outside the AuNR surfaces. The insets in Figure S9 show an enhancement factor of 4 orders of magnitude in the near-field intensity at the gap regions. In contrast, the field enhancement near the rest of the rod surfaces, including the outer rod ends of the arrays, is orders of magnitude smaller, and despite the larger area in those regions, we expect their contribution to be comparatively negligible. We therefore anticipate that effects associated with molecules placed on AuNR surfaces will be largely dominated by those lying within internanorod gap regions. The enhancement magnitude seems to be similar in all gaps, regardless of the number of rods in the structure. However, this number, possibly together with the size distribution of AuNRs, determines the light wavelength at which enhancement will be maximum in the gaps. Interestingly, all gaps within a given structure present maximum enhancement at a common wavelength (Figure S10). It should be noted that higher-order resonances besides the main dipolar mode of the coupled structure are not efficiently excited for the array sizes under consideration, even under oblique light incidence (Figure S11).

We now relate the near field to the CDLE by relying on general properties of the chiral response of molecules, combined with the reciprocity theorem to connect near- and far-field amplitudes in illuminated nanostructures. Application of these principles allows us to define the CDLE (see Methods for a detailed derivation) as

$$
\operatorname{CDLE}(\mathbf{r}) \equiv \frac{1}{4 n\left|E_{0}\right|^{2}} \sum_{v} s_{v}\left[\mathbf{E}_{v}(\mathbf{r},-\hat{\mathbf{k}}) \cdot \mathbf{H}_{v}(\mathbf{r}, \hat{\mathbf{k}})+\mathbf{H}_{v}(\mathbf{r},-\hat{\mathbf{k}}) \cdot \mathbf{E}_{v}(\mathbf{r}, \hat{\mathbf{k}})\right]
$$

where $\mathbf{E}$ and $\mathbf{H}$ are the electric and magnetic field amplitudes acting on the molecule, $E_{0}$ is the incident-light field amplitude, $n$ is the refractive index of the solvent, $\mathbf{r}$ is the position of the molecule, and we define $s_{\mathrm{L}}=1$ and $s_{\mathrm{R}}=-1$ for $v=\mathrm{L}(\mathrm{LCP})$ and $v=\mathrm{R}$ (RCP) light polarization. We expect that eq 1 provides a good estimate of the CD enhancement using as input just the optical 
near field obtained from electromagnetic calculations for $v=\mathrm{L}$ and $v=\mathrm{R}$ light polarization incident from the direction of the source in the experiment ( $\hat{\mathbf{k}}$ propagation direction) and from the detector (- $\mathbf{k}$ direction). This formalism allows us to map the enhancement in the contribution to $\mathrm{CD}$ due to chiral molecules as a function of their position in the vicinity of the AuNRs. The results are summarized in Figure 4, where the CD enhancement is normalized relative to the contribution by molecules far away from the structures. The results, which show a CDLE that essentially follows the same trends as the near-field enhancement (cf. Figure $\mathbf{4}$ and the near-field insets of Figure S9), are in good agreement with our experimental data, wherein we observed a dramatic increase in the plasmonic CD during the linear assembly of AuNRs. The enhancement in the CD signal is thus produced by the generation of strong fields at the linear junctions (hotspots) and the entrapment of chiral molecules at these hotspots.

We remark that the observed CD signal in the plasmonic region does not originate from a helicitydependence of molecular absorption: molecules are not effectively absorbing in this region. Instead, we attribute the CD signal to a purely elastic effect, in which the optically-active refractive index rather than absorption depends on light helicity (see discussion at the end of the Methods section for more details). This nonabsorbing source of a dichroism signal is indeed observed in the 600 nm signal of dispersed molecules without AuNRs (see above), so the effect of plasmonic hotspots in aggregated particles is to amplify the CD signal. 



Figure 4. Calculated enhancement in the contribution to $C D$ as a function of the position of chiral molecules. (a) Spatial distribution of CDLE for individual $(N=1)$ and linearly-arrayed $(N=2-$ 4) AuNRs. (b) Spectral dependence of CDLE averaged over the surface of the $N=2$ structure.

\section{CONCLUSIONS}

In summary, we have demonstrated the enhancement of the optical chirality signal emanating from a small amount of molecules in the visible and near-IR ranges due to exposure to plasmonmediated optical hotpots. Judicious design of molecular entities allowed us to control the assembly of AuNRs, thereby enhancing optical activity over the plasmonic spectral window. Our results, which rely on a general method for directing nanorod assembly, do not imply a specific interaction of the molecule with the nanorod surface. Instead, they suggest an alternative hypothesis for the origin of geometrically selective association of nanoparticles, based on surfactant cap destabilization, thereby contributing to clarify the still complex scenario of nanorod assembly 
process. The intrinsically weak chiral vis-NIR signal from chiral molecules could be enhanced due to the formation of plasmonic hotspots generated by the preferential tip-to-tip self-assembly of AuNRs. The linear assemblies generate enhanced electric fields at the gaps between particle tips, which give rise to amplification of the chirality induced by co-surfactants. These results unequivocally link the observation of plasmonic CD signals to enhancement of the primary CD signal from the chiral linker molecules located within the plasmonic hotspots in the metal nanoparticles. Our experimental observations were explained by using a theoretical description of plasmon-enhanced chirality that could find further application in the design of optimized particle morphologies and arrangements, to achieve maximum amplification of molecular $\mathrm{CD}$ and therefore reduce the molecular density required for experimental detection. The design strategy adopted herein suggests that surfactants can function as efficient tools to induce enhanced signals in biosensing platforms based on plasmonic nanoparticles.

\section{METHODS}

General Experimental Procedures. Hydrogen tetrachloroaurate trihydrate $\left(\mathrm{HAuCl}_{4} \cdot 3 \mathrm{H}_{2} \mathrm{O}, \geq\right.$ 99.9\%), СТАВ (99\%), silver nitrate $\left(\mathrm{AgNO}_{3}, \geq 99.0 \%\right)$, L-ascorbic acid ( $\left.\geq 99 \%\right)$, sodium borohydride $\left(\mathrm{NaBH}_{4}, 99 \%\right)$, sodium cholate $(\geq 99 \%)$, glycocholate $(\geq 95 \%)$, and taurocholate $(\geq$ 97\%) were purchased from Sigma-Aldrich and were used without further purification. Milli-Q water was used in all experiments.

Extinction spectra were measured using an Agilent 8453 UV-visible diode-array spectrophotometer. CD measurements were performed on Jasco J-815 and J-1500 CD spectrophotometers. Although CD measurements can suffer from artefacts due to linear dichroism (LD), the nanorods here were randomly orientated due to their free-floating nature in solution. To further rule out the possibility of preferential alignment of the nanorods, control experiments were performed at different orientations by rotating the cuvette by 90 degrees. The resulting spectra exhibited no significant differences, ruling out any possibility of LD contributions. Transmission electron microscopy (TEM) images were collected on a JEOL JEM-2100F electron microscope, 
with an acceleration voltage of $200 \mathrm{kV}$. TEM samples were prepared by adding a few drops of the nanorod assemblies at different stages of aggregation (5, 10, and 15 min) onto a carbon coated copper grid placed on a filter paper. The grid was dried overnight at room temperature before TEM analysis. Image $\mathrm{J}$ software was used to calculate the dimensions of nanorods from TEM images. About 100-200 nanorods per sample were analyzed. DLS and electrophoretic mobility $(\mu)$ measurements were performed with a Malvern Nano-ZetaSizer. The instrument was equipped with a digital logarithmic correlator and a $5 \mathrm{~mW}$ HeNe laser $(\lambda=632.8 \mathrm{~nm})$. The normalized intensity autocorrelation function was measured at an angle of $173^{\circ}$, at $25.0 \pm 0.1^{\circ} \mathrm{C}$, and analyzed by using the cumulant fit method to provide the apparent hydrodynamic diameter $\left(\mathrm{D}_{\mathrm{H}}\right)$. Laser Doppler electrophoresis was employed to obtain the electrophoretic mobility, by analyzing the Doppler shift in the Zetasizer Nano, according to the phase analysis light scattering (PALS) technique. The values reported in this manuscript were averaged over three consecutive measurements for each sample. SEM measurements were performed by a HR-FESEM AURIGA ZEISS instrument with an In-Lens detector, operating at $18.00 \mathrm{kV}$. The samples were prepared by depositing a drop of solution on a TEM copper grid covered with a thin amorphous carbon film. Excess liquid was removed with filter paper. Specific optical rotations $[\alpha]_{\mathrm{D}}\left(\mathrm{deg} \cdot \mathrm{mL} \cdot \mathrm{g}^{-1} \cdot \mathrm{dm}^{-1}\right)$ were measured at a wavelength of $589.3 \mathrm{~nm}$ and $25{ }^{\circ} \mathrm{C}$ with a digital polarimeter (Jasco DIP-370) by using a path length of $1 \mathrm{~cm}$ on solutions of $3.74 \mathrm{mg} / \mathrm{mL} \mathrm{NaTPC}$ in methanol and $15.0 \mathrm{mg} / \mathrm{mL}$ sodium cholate in water.

Synthesis of NaTPC. The synthesis of the bile salt derivative NaTPC was reported elsewhere. ${ }^{41}$ In brief, cholic acid was first converted into a cholic acid ester, and then transformed into the corresponding $3 \beta$-amino substituted derivative via the Mitsunobu reaction, with diphenylphosphoril azide, followed by Staudinger reduction, with $\mathrm{PPh}_{3} / \mathrm{H}_{2} \mathrm{O}$ of the intermediate azido compound in THF. ${ }^{42}$ NaTPC was obtained by reacting $p$-tertbutyl benzoyl chloride with the 3 $\beta$-amino substituted cholic ester, which was then hydrolyzed.

AuNR synthesis following the seedless growth method. AuNRs with a longitudinal LSPR at 815 $\mathrm{nm}$ were synthesized using the seedless growth method previously reported by El-Sayed and coworkers, with some modifications. ${ }^{28}$ To a solution of $\mathrm{HAuCl}_{4}(100 \mu \mathrm{L}, 0.05 \mathrm{M})$ in CTAB $(10.0$ $\mathrm{mL}, 0.1 \mathrm{M}), 250 \mu \mathrm{L}$ of $\mathrm{AgNO}_{3}(4.0 \mathrm{mM})$ was added and gently shaken. $\mathrm{HCl}(12.0 \mu \mathrm{L}, 37 \mathrm{wt} \%)$ 
was introduced to attain a pH close to 1.0. Subsequently, $70 \mu \mathrm{L}$ of ascorbic acid (78.8 mM) was added under gentle stirring until the solution became clear. Finally, ice-cold $\mathrm{NaBH}_{4}(10 \mu \mathrm{L}, 0.01$ M) was injected into the growth solution and allowed to react for $6 \mathrm{~h}$. The synthesized NR solution was centrifuged twice at $14500 \mathrm{rpm}$ to remove excess of CTAB and other reagents. The supernatant was discarded and the NRs settled at the bottom of the centrifuge tube were redispersed in water and used for further studies.

AuNR synthesis following the seed-mediated method. AuNRs with a longitudinal LSPR at 685 nm were synthesized using the seed-mediated growth method reported by Nikoobakht and ElSayed, ${ }^{43}$ involving preparation of seeds and NR synthesis:

- $\quad$ Preparation of seeds: $\mathrm{HAuCl}_{4}(5.0 \mathrm{~mL}, 0.00050 \mathrm{M})$ was added to CTAB solution (5 mL, $0.20 \mathrm{M})$ and stirred. To the stirring solution, ice-cold $\mathrm{NaBH}_{4}(0.60 \mathrm{~mL}, 0.010 \mathrm{M})$ was added resulting in the formation of a brownish yellow solution. Vigorous stirring was continued for 2 min and the resultant seed solution was stored at $25{ }^{\circ} \mathrm{C}$.

- $\quad$ Synthesis of nanorods: $\mathrm{AgNO}_{3}(0.0040 \mathrm{M}, 0.20)$ was added to CTAB (5 mL, $\left.0.20 \mathrm{M}\right)$ solution at $25{ }^{\circ} \mathrm{C}$. To this mixture, $\mathrm{HAuCl}_{4}(5.0 \mathrm{~mL}, 0.0010 \mathrm{M})$ was added and gently mixed. Thereafter, a mild reducing agent, ascorbic acid $(70 \mathrm{~mL}, 0.0788 \mathrm{M})$, was added, resulting in a colorless solution. In the final step, $12 \mu \mathrm{L}$ of the seed was added to the growth solution at $27^{\circ} \mathrm{C}$. The color of the solution gradually changed within 10-20 min, confirming the formation of AuNRs. The solution was centrifuged twice at $8000 \mathrm{rpm}$ for $20 \mathrm{~min}$ to remove excess CTAB and other reagents.

Self-assembly of AuNRs. NaTPC was added in a step-wise manner to AuNRs dispersed in water. Extinction spectra were monitored after every single addition. The first addition of NaTPC resulted in a bathochromic shift in the longitudinal LSPR band due to the change in refractive index of the medium. Subsequent additions resulted in no noticeable changes in the extinction spectral features until the concentration reached a critical value wherein a gradual decrease in the longitudinal LSPR band was observed, with the formation of an emerging band in the NIR region. Spectral shifts saturated at $20 \mathrm{~min}$ after the addition of $25 \mu \mathrm{M}$ of NaTPC to $1.2 \mathrm{nM}$ of AuNRs (for AuNRs with a longitudinal LSPR of $815 \mathrm{~nm}$ ) in water. CD spectra of the samples were measured upon saturation of the spectral changes. Additional CD spectra were collected after each addition of the 
co-surfactant for concentration-dependent analysis (Figure S2). TEM samples were prepared at different stages of the assembly process. For this, samples were drawn out of the solution at varying time intervals (5, 10, and $15 \mathrm{~min})$ and drop-casted onto a carbon coated copper grid. Similar experiments were performed using SDS and other bile salt derivatives as the co-surfactants.

\section{Theoretical Simulations for Circular Dichroism of Chiral Molecules in Plasmonic Hostpots.}

Chiral molecules present an optical response that can be described through their $6 \times 6$ polarizability tensor

$$
\boldsymbol{\alpha}=\left(\begin{array}{cc}
\boldsymbol{\alpha}_{\mathrm{EE}} & \boldsymbol{\alpha}_{\mathrm{EM}} \\
\boldsymbol{\alpha}_{\mathrm{ME}} & \boldsymbol{\alpha}_{\mathrm{MM}}
\end{array}\right)
$$

implicitly defined through the relation $\left(\begin{array}{l}\mathbf{p} \\ \mathbf{m}\end{array}\right)=\boldsymbol{\alpha} \cdot\left(\begin{array}{l}\mathbf{E} \\ \mathbf{H}\end{array}\right)$ between the electric and magnetic field amplitudes $\mathbf{E}$ and $\mathbf{H}$ acting on the molecule and the induced electric and magnetic dipoles $\mathbf{p}$ and $\mathbf{m}$, respectively. The contribution of different optical resonances to the components of eq 2 ( $\boldsymbol{\alpha}_{j j}$, which are $3 \times 3$ matrices that connect electric $(j=\mathrm{E})$ and magnetic $(j=\mathrm{M})$ parts) can be expressed in terms of the corresponding transition matrix elements. In particular, chirality is associated with the presence of a nonzero electric-magnetic component $\boldsymbol{\alpha}_{\mathrm{EM}}$, which originates in the simultaneous existence of nonparallel electric and magnetic transition dipoles in the resonances. $^{31,44}$ Incidentally, reciprocity implies that $\boldsymbol{\alpha}_{\mathrm{ME}}=-\boldsymbol{\alpha}_{\mathrm{EM}}^{\mathrm{T}}$, where the superscript $\mathrm{T}$ stands for the transpose.

For molecules in solution, the average over molecular orientations results in a mean polarizability such that $\boldsymbol{\alpha}_{j j^{\prime}}=\alpha_{j j^{\prime}} \mathbb{I}$, where $\alpha_{j j^{\prime}}$ are complex numbers and II is the $3 \times 3$ identity matrix. In CGS units, the electric and magnetic fields for a circularly-polarized (CP) light plane wave propagating along the positive $\hat{\mathbf{z}}$ direction $\operatorname{are}^{45} \mathbf{E}=\left(E_{0} / \sqrt{2}\right)(\hat{\mathbf{x}} \pm i \hat{\mathbf{y}})$ and $\mathbf{H}=$ $\mp i\left(n E_{0} / \sqrt{2}\right)(\hat{\mathbf{x}} \pm i \hat{\mathbf{y}})$, where upper and lower signs correspond to left and right CP light (from the point of view of the light detector), respectively, $E_{0}$ denotes the electric field amplitude, and $n$ is the refractive index of the solvent. The induced dipoles then reduce to $\mathbf{p}=\left(E_{0} / \sqrt{2}\right)(\hat{\mathbf{x}} \pm$ $i \hat{\mathbf{y}})\left(\alpha_{\mathrm{EE}} \mp i n \alpha_{\mathrm{EM}}\right)$ and $\mathbf{m}=\left(E_{0} / \sqrt{2}\right)(\hat{\mathbf{x}} \pm i \hat{\mathbf{y}})\left(-\alpha_{\mathrm{EM}} \mp i n \alpha_{\mathrm{MM}}\right)$, which result in a forward electric far-field scattering amplitude $\mathbf{f}_{\mathrm{E}}=\left(k^{2} E_{0} / \sqrt{2}\right)(\hat{\mathbf{x}} \pm i \hat{\mathbf{y}})\left(\alpha_{\mathrm{EE}}+n^{2} \alpha_{\mathrm{MM}} \mp 2 n i \alpha_{\mathrm{EM}}\right)$, where $k=\omega / c$ is the free-space light wave vector for light of frequency $\omega$. Now, the extinction 
cross section, which is a quantity directly accessible in optical transmission experiments, is given by $^{46}$

$$
\sigma^{\mathrm{ext}}=\frac{4 \pi}{k n\left|E_{0}\right|^{2}} \operatorname{Im}\left\{\mathbf{f}_{\mathrm{E}} \cdot \mathbf{E}^{*}\right\}
$$

After propagation through a dispersion, the ratio of transmitted to incident intensity is simply given by $I / I_{0}=\exp \left(-\rho L \sigma^{\text {ext }}\right)$, or equivalently $\sigma^{\text {ext }}=(1 / \rho L) \ln \left(I_{0} / I\right)$, where $\rho$ is the molecular density and $L$ the optical path length in the solution. Direct application of eq 3 leads to the following expression for the CD signal:

$$
\mathrm{CD}=\frac{\sigma_{\mathrm{L}}^{\text {ext }}-\sigma_{\mathrm{R}}^{\text {ext }}}{\sigma_{\mathrm{L}}^{\text {ext }}+\sigma_{\mathrm{R}}^{\text {ext }}}=-2 n \frac{\operatorname{Re}\left\{\alpha_{\mathrm{EM}}\right\}}{\operatorname{Im}\left\{\alpha_{\mathrm{EE}}+n^{2} \alpha_{\mathrm{MM}}\right\}} \approx-2 n \frac{\operatorname{Re}\left\{\alpha_{\mathrm{EM}}\right\}}{\operatorname{Im}\left\{\alpha_{\mathrm{EE}}\right\}}
$$

where the sub-indices $\mathrm{L}$ and $\mathrm{R}$ refer to left and right CP light, and the rightmost approximation takes advantage of the fact that $\left|\alpha_{\mathrm{MM}}\right| \ll\left|\alpha_{\mathrm{EE}}\right|$. Incidentally, for a small molecular volume density $n_{\mathrm{V}}$ in the solution, the light wave vector becomes $k_{ \pm}=k \sqrt{\left(1+4 \pi k n_{\mathrm{V}} \alpha_{\mathrm{EE}}\right)\left(1+4 \pi k n_{\mathrm{V}} \alpha_{\mathrm{MM}}\right)} \mp$ $4 \pi i k n_{\mathrm{V}} \alpha_{\mathrm{EM}}$ for left (upper sign) and right (lower sign) circularly polarized light (i.e., the field amplitude depends on propagation distance $z$ as $e^{i k_{ \pm} z}$ ), clearly revealing that $\operatorname{Re}\left\{\alpha_{\mathrm{EM}}\right\}$ affects the inelastic attenuation distance, whereas $\operatorname{Im}\left\{\alpha_{\mathrm{EM}}\right\}$ contributes to the propagation phase. CD in homogeneous solution is thus related to a difference in absorption depending on light helicity.

The CD signal can depend on the details of the near field when the molecules are placed close to nanostructures. In order to maintain the discussion sufficiently general for cases in which the molecules do not have a preferential orientation relative to the surface, we describe them in what follows through a polarizability like that in eq 2 , with the four $3 \times 3$ components assumed to be diagonal just like in the homogeneous solution discussed above.

We are interested in describing the CD signal produced by chiral molecules placed in the vicinity of metallic nanorods. Specifically, we intend to quantify the increase in CD produced by the near-field enhancement associated with plasmons sustained by arrayed particles. We here introduce a general theory capable of describing effects produced by the presence of molecules in plasmonic structures under the assumption that their density is small and their interaction with the structures is weak enough to be described to first order. As magnetic dipoles are relevant in the description of CD (see above), we use for simplicity a compact notation that combines electric and magnetic quantities together: 


$$
\mathbf{d}=\left(\begin{array}{c}
\mathbf{p} \\
\mathbf{m}
\end{array}\right), \quad \mathbf{F}=\left(\begin{array}{c}
\mathbf{E} \\
\mathbf{H}
\end{array}\right)
$$

which denote an electric dipole $\mathbf{p}$ and a magnetic dipole $\mathbf{m}$ through $\mathbf{d}$, as well as an electric field amplitude $\mathbf{E}$ and a magnetic field amplitude $\mathbf{H}$ through $\mathbf{F}$. Without loss of generality, we describe the light source as a set of far-field emitting dipoles $\mathbf{d}_{\mathbf{s}}$ at positions $\mathbf{r}_{\mathrm{s}}$, while the molecules are distributed at positions $\mathbf{r}_{i}$ and represented through their induced dipoles $\mathbf{d}_{i}$. We are interested in evaluating the resulting far-field electric field at the detector position $\mathbf{r}_{\mathrm{d}}$. The interactions among different elements can be rigorously described through the $6 \times 6$ electromagnetic Green tensor $\mathcal{G}\left(\mathbf{r}, \mathbf{r}^{\prime}, \omega\right)$, which can be defined in simple terms as the quantity that allows us to calculate the electromagnetic field $\mathbf{F}(\mathbf{r})$ produced at a position $\mathbf{r}$ by a dipole $\mathbf{d}$ placed at $\mathbf{r}^{\prime}$, that is,

$$
\mathbf{F}(\mathbf{r})=\mathcal{G}\left(\mathbf{r}, \mathbf{r}^{\prime}, \omega\right) \cdot \mathbf{d}
$$

where we assume monochromatic light of frequency $\omega$, so that the time-dependent electromagnetic field is given by $\mathbf{F}(\mathbf{r}, t)=2 \operatorname{Re}\left\{\mathbf{F}(\mathbf{r}) e^{-i \omega t}\right\}$, and similarly for other electromagnetic quantities. We further introduce the $6 \times 6$ molecular polarizability tensor $\boldsymbol{\alpha}_{i}$, which relates the field acting on the molecule at $\mathbf{r}_{i}$ to the induced dipole through

$$
\mathbf{d}_{i}=\boldsymbol{\alpha}_{i} \cdot \mathbf{F}\left(\mathbf{r}_{i}\right)
$$

(i.e., a compact version of eq 2). For a well collimated light source, the incident field illuminating the structure and also reaching the detector is a plane wave $\sum_{s} \mathcal{G}^{0}\left(\mathbf{r}, \mathbf{r}_{\mathrm{s}}, \omega\right) \cdot \mathbf{d}_{\mathrm{s}}=\mathbf{F}^{0} e^{i \mathbf{k}^{0} \cdot \mathbf{r}}$, where $\mathcal{G}^{0}$ is the Green tensor of the homogeneous solvent environment and we integrate over source dipole positions $\mathbf{r}_{\mathrm{s}}$ (see Figure S12). The presence of the particles adds a scattering component to the Green tensor, which can thus be decomposed as $\mathcal{G}=\mathcal{G}^{0}+\mathcal{G}^{\text {scat }}$. Without molecules, the field at the detector is then the sum of incident and scattered contributions,

$$
\sum_{s} \mathcal{G}\left(\mathbf{r}_{\mathrm{d}}, \mathbf{r}_{\mathrm{s}}, \omega\right) \cdot \mathbf{d}_{\mathrm{s}}=\mathbf{F}^{0} e^{i \mathbf{k}^{0} \cdot \mathbf{r}_{\mathrm{d}}}+\sum_{s} \mathcal{G}^{\mathrm{scat}}\left(\mathbf{r}_{\mathrm{d}}, \mathbf{r}_{\mathrm{s}}, \omega\right) \cdot \mathbf{d}_{\mathrm{s}} \approx \mathbf{F}^{0} e^{i \mathbf{k}^{0} \cdot \mathbf{r}_{\mathrm{d}}}+\mathbf{f}^{\mathrm{scat}}\left(\hat{\mathbf{r}}_{\mathrm{d}}\right) \frac{e^{i k n r_{\mathrm{d}}}}{r_{\mathrm{d}}}
$$

where the rightmost approximation is valid in the far-field limit $k n r_{\mathrm{d}} \gg 1$ and $\mathbf{f}^{\mathrm{scat}}\left(\hat{\mathbf{r}}_{\mathrm{d}}\right)$ is a scattering amplitude that depends on the scattering direction $\widehat{\mathbf{r}}_{\mathrm{d}}$. Now, the incident and scattered fields enter the extinction cross section through a factor ${ }^{45} \operatorname{Im}\left\{\left(\mathbf{E}^{0}\right)^{*} \cdot \mathbf{f}_{\mathrm{E}}^{\text {scat }}\left(\hat{\mathbf{r}}_{\mathrm{d}}\right)\right\}$ (see eq 3 ), where $\mathbf{f}_{\mathrm{E}}^{\text {scat }}$ denotes the electric part of the scattering amplitude. This result is rigorous, regardless of the 
size and nature of the scattering system. ${ }^{47}$ We note that the polarization of the incident light is fully encoded in the complex components of the incident electric field amplitude $\mathbf{E}^{0}$.

For individual particles and linear assemblies of nanorods dispersed in solution without molecules, the extinction signal consists of an average over realizations and orientations of those structures. The signal is then polarization-independent (i.e., no circular dichroism) because no net chirality is preserved after such averaging if the particles or their arrangements are not chiral. However, the presence of chiral molecules on the particle surfaces adds a dependence on polarization because the chirality of the molecules remains unchanged even after averaging over rotations of the system; this is similar to the analysis presented above for molecules in a homogeneous solution. In order to analyze the effect of the molecules present on the particle surfaces, we note that the Green tensor of the combined system (particles plus molecules) $\mathcal{G}^{\text {total }}$ contains additional components produced by molecular scattering. Using the definitions of eqs 4 and 5, we can write, neglecting multiple molecular scattering,

$$
\mathcal{G}^{\text {total }}\left(\mathbf{r}_{\mathrm{d}}, \mathbf{r}_{\mathrm{s}}, \omega\right)=\mathcal{G}\left(\mathbf{r}_{\mathrm{d}}, \mathbf{r}_{\mathrm{s}}, \omega\right)+\sum_{i} \mathcal{G}\left(\mathbf{r}_{\mathrm{d}}, \mathbf{r}_{i}, \omega\right) \cdot \boldsymbol{\alpha}_{i} \cdot \mathcal{G}\left(\mathbf{r}_{i}, \mathbf{r}_{\mathrm{s}}, \omega\right)
$$

where the second term describes light propagation from the source to each of the molecules $(i$ label), combined with the molecular polarizability to obtain the induced dipole, and followed by subsequent propagation of the light scattered by the dipole to the detector. We now use the reciprocity theorem (i.e., $\mathcal{G}_{\mathrm{EE}, a b}\left(\mathbf{r}, \mathbf{r}^{\prime}, \omega\right)=\mathcal{G}_{\mathrm{EE}, b a}\left(\mathbf{r}^{\prime}, \mathbf{r}, \omega\right)$ and $\mathcal{G}_{\mathrm{EM}, a b}\left(\mathbf{r}, \mathbf{r}^{\prime}, \omega\right)=-\mathcal{G}_{\mathrm{ME}, b a}\left(\mathbf{r}^{\prime}, \mathbf{r}, \omega\right)$, where $a$ and $b$ denote Cartesian components) and recall the fact that the direct source field amplitude is uniform over the sample under collimated irradiation conditions. This allows us to write from eqs 4 and 6 the following expression for the change in electric scattering amplitude due to the molecules:

$$
\Delta \mathbf{f}_{\mathrm{E}}^{\text {scat }}\left(\hat{\mathbf{r}}_{\mathrm{d}}\right) \propto \sum_{i, v} \hat{\boldsymbol{\varepsilon}}_{v}\left[\mathbf{F}_{v}^{\text {out,S }}\left(\mathbf{r}_{i}\right) \cdot \boldsymbol{\alpha}_{i} \cdot \mathbf{F}^{\text {in }}\left(\mathbf{r}_{i}\right)\right]
$$

where $v$ runs over a complete set of orthogonal polarizations associated with unit vectors $\hat{\boldsymbol{\varepsilon}}_{v}=$ $(\hat{\mathbf{x}} \pm i \hat{\mathbf{y}}) / \sqrt{2}$, with upper and lower signs standing for $v=\mathrm{L}$ and $v=\mathrm{R}$, respectively; $\mathbf{F}^{\mathrm{in}}\left(\mathbf{r}_{i}\right)$ is the near field acting on molecule $i$ as produced upon external illumination in the presence of the structure but without any of the molecules; and $\mathbf{F}_{v}^{\text {out,S }}\left(\mathbf{r}_{i}\right)$ is the near field generated under irradiation with reverse propagation (i.e., coming instead from the detector), with the superscript $\mathrm{S}$ indicating that the sign of the magnetic field is flipped. The above expression, which contains 
the whole dependence on $\boldsymbol{\alpha}_{i}$, omits an irrelevant geometry- and frequency-dependent real-number factor that is independent of the molecular positions and polarizabilities. Using eq 7, we find the change in extinction cross section due to the molecules to be $\Delta \sigma^{\text {ext }} \propto \operatorname{Im}\left\{\mathbf{E}_{\mathrm{s}}^{*} \cdot \Delta \mathbf{f}^{\text {scat }}\left(\hat{\mathbf{r}}_{\mathrm{d}}\right)\right\} \propto$ $\sum_{i, v} \operatorname{Im}\left\{\mathbf{E}_{\mathbf{s}}^{*} \cdot \hat{\boldsymbol{\varepsilon}}_{v}\left[\mathbf{F}_{v}^{\text {out,S }}\left(\mathbf{r}_{i}\right) \cdot \boldsymbol{\alpha}_{i} \cdot \mathbf{F}^{\text {in }}\left(\mathbf{r}_{i}\right)\right]\right\}$. Now, it is convenient to introduce the notation $\mathbf{F}_{v}(\mathbf{r}, \hat{\mathbf{k}})$ to refer to the near field produced at point $\mathbf{r}$ upon illumination by a plane wave of polarization $v$ propagating along the direction $\hat{\mathbf{k}}$. This allows us to write the change produced by the presence of the molecules in the extinction cross section for incident polarization $v$ (i.e., $\mathbf{E}_{\mathrm{s}} \propto \hat{\boldsymbol{\varepsilon}}_{v}$ ) as $\Delta \sigma_{v}^{\text {ext }} \propto$ $\sum_{i} \operatorname{Im}\left\{\mathbf{F}_{v}^{\mathrm{S}}\left(\mathbf{r}_{i},-\hat{\mathbf{k}}\right) \cdot \boldsymbol{\alpha}_{i} \cdot \mathbf{F}_{v}\left(\mathbf{r}_{i}, \hat{\mathbf{k}}\right)\right\}$, which leads to a CD signal

$$
\begin{gathered}
\mathrm{CD}=\frac{\sigma_{\mathrm{L}}^{\mathrm{ext}}-\sigma_{\mathrm{R}}^{\mathrm{ext}}}{\sigma_{\mathrm{L}}^{\mathrm{ext}}+\sigma_{\mathrm{R}}^{\mathrm{ext}}} \approx \frac{\Delta \sigma_{\mathrm{L}}^{\mathrm{ext}}-\Delta \sigma_{\mathrm{R}}^{\mathrm{ext}}}{\sigma^{\mathrm{ext}}} \\
\approx \frac{4 \pi}{\sigma^{\mathrm{ext}} k n\left|E_{0}\right|^{2}} \operatorname{Im}\left\{\alpha_{\mathrm{EM}} \sum_{i, v} s_{v}\left[\mathbf{E}_{v}\left(\mathbf{r}_{i},-\hat{\mathbf{k}}\right) \cdot \mathbf{H}_{v}\left(\mathbf{r}_{i}, \hat{\mathbf{k}}\right)+\mathbf{H}_{v}\left(\mathbf{r}_{i},-\hat{\mathbf{k}}\right) \cdot \mathbf{E}_{v}\left(\mathbf{r}_{i}, \hat{\mathbf{k}}\right)\right]\right\},
\end{gathered}
$$

where $s_{v}=1$ for LCP and -1 for RCP light, and $E_{0}$ is the incident electric field amplitude. In the derivation of this expression, we have exploited the fact that the change in cross section is small compared with the total cross section (i.e., $\left|\Delta \sigma_{v}^{\text {ext }}\right| \ll \sigma_{v}^{\text {ext }} \approx \sigma^{\text {ext }}$, where $\sigma^{\text {ext }}$ is the polarizationaveraged cross section) and also $\left|\alpha_{\mathrm{MM}}\right|,\left|\alpha_{\mathrm{EM}}\right| \ll\left|\alpha_{\mathrm{EE}}\right|$ (see above). Equation 8 gives the magnitude of the CD signal in the presence of near-field enhancement and it needs to be compared with the result for a homogeneous solution, $-2 n \operatorname{Re}\left\{\alpha_{\mathrm{EM}}\right\} / \operatorname{Im}\left\{\alpha_{\mathrm{EE}}\right\}$. In order to present an analysis that bears general validity for any type of molecule and structure, we dismiss the phase in $\alpha_{\mathrm{EM}}$ and focus on the near-field enhancement quantified in the $\mathbf{E}_{v}$ and $\mathbf{H}_{v}$ fields. We thus consider the combined near-field factor in eq 8, which allows us to define the CDLE as shown in eq 1 in the main text, yielding $\mathrm{CD} \approx\left(16 \pi / \sigma^{\text {ext }} k\right) \operatorname{Im}\left\{\alpha_{\mathrm{EM}} \sum_{i} \operatorname{CDLE}\left(\mathbf{r}_{i}\right)\right\}$. We note that eq 1 is normalized to yield a value $\operatorname{CDLE}(\mathbf{r})=1$ for $\mathbf{r}$ located far from the particles in the solvent. Additionally, eq 1 ignores the phase of $\alpha_{\mathrm{EM}}$, which affects the CD signal in relation to the phases of both the near field the diagonal polarizability $\alpha_{\mathrm{EE}}$; a more detailed analysis would require knowledge of the spectral dependence of the molecular polarizability (including electric-magnetic crossed terms), as well as a complex averaging over particle orientations and arrangements. However, we expect that eq 1 provides a good estimate of the CD enhancement from straightforward electromagnetic calculations of the near-field obtained for $v=\mathrm{L}$ (LCP) and $v=\mathrm{R}$ (RCP) incidence from the source

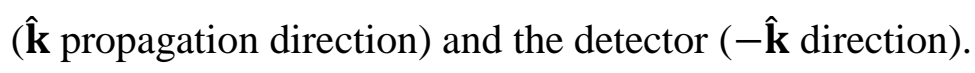


Molecules display chiral transitions at UV frequencies $\omega_{j}$, each of them characterized by nonorthogonal electric and magnetic dipoles $\mathbf{p}_{j}$ and $\mathbf{m}_{j}$, respectively, which give rise to contributions to the polarizability tensor with a frequency dependence ${ }^{48} \alpha_{\mathrm{EE}} \propto\left(p_{j}^{2} / \hbar\right) /\left(\omega_{j}-\right.$ $\left.\omega-i \gamma_{j} / 2\right), \quad \alpha_{\mathrm{MM}} \propto\left(m_{j}^{2} / \hbar\right) /\left(\omega_{j}-\omega-i \gamma_{j} / 2\right), \quad$ and $\quad \alpha_{\mathrm{MM}} \propto\left(p_{j} m_{j} / \hbar\right) /\left(\omega_{j}-\omega-i \gamma_{j} / 2\right)$, where $\gamma_{j}$ is the resonance width. Now, $p_{j}$ and $m_{j}$ are real and imaginary numbers, respectively, ${ }^{44}$ so we deduce that absorptive components $\left(\operatorname{Im}\left\{\alpha_{\mathrm{EE}}\right\}, \operatorname{Im}\left\{\alpha_{\mathrm{MM}}\right\}\right.$, and $\left.\operatorname{Re}\left\{\alpha_{\mathrm{EM}}\right\}\right)$ produce sharp spectral absorption features $\propto 1 /\left|\omega_{j}-\omega-i \gamma_{j} / 2\right|^{2}$ (i.e., they die out as the inverse square of the frequency detuning outside the FWHM spectral width $\gamma_{j}$ ); in contrast, the remaining refractive (nonabsorbing) components $\left(\operatorname{Re}\left\{\alpha_{\mathrm{EE}}\right\}, \operatorname{Re}\left\{\alpha_{\mathrm{MM}}\right\}\right.$, and $\left.\operatorname{Im}\left\{\alpha_{\mathrm{EM}}\right\}\right)$ exhibit a weaker spectral decay as $\propto 1 /\left(\omega_{j}-\omega\right)$ away from the resonance. This explains why UV resonances contribute to change the refractive index in the visible (also in virtue of the Kramers-Kronig relation), while in the present context we conclude that $\operatorname{Im}\left\{\alpha_{\mathrm{EM}}\right\}$ can take significant off-resonance values that contribute to eq 8 in the visible-NIR plasmonic region. This argument indicates that our observation of chirality has a refractive rather than absorptive origin, whereby the nonresonant, nonabsorbing, refractive component $\operatorname{Im}\left\{\alpha_{\mathrm{EM}}\right\}$ of the molecular chiral polarizability does transform into a measurable change in the plasmonic extinction at visible-NIR frequencies through nonresonant molecule-plasmon coupling that is effectively described by eq 8, therefore anticipating a $\mathrm{CD} \sim \operatorname{Im}\left\{\alpha_{\mathrm{EM}}\right\} \operatorname{Re}\{\mathrm{CDLE}\}$ signal (see Figure $4 \mathrm{~b}$ ). In summary, a CD signal (i.e., a helicitydependent difference in optical absorption) is produced in a homogeneous molecular solution (i.e., in the absence of plasmonic nanoparticles) that is roughly proportional to $\operatorname{Re}\left\{\alpha_{\mathrm{EM}}\right\}$ and emerges as sharp resonance features in the UV, whereas a helicity-dependent propagation phase is introduced thought $\propto \operatorname{Im}\left\{\alpha_{\mathrm{EM}}\right\}$, which still takes small, but non-negligible values in the visibleNIR, far from the UV resonances. In the presence of nanoparticles, the latter is enhanced by coupling to plasmonic absorption, thus resulting in a strong CD signal driven by optical absorption in the nanoparticles mediated by the nonabsorbing molecular polarizability component $\propto$ $\operatorname{Im}\left\{\alpha_{\mathrm{EM}}\right\}$.

\section{ASSOCIATED CONTENT}

\section{Supporting Information}


Statistical analysis of contacts in assemblies of nanorods, optical response of nanorods with different aspect ratio, nanorod self-assembly induced by SDS and natural bile salts, estimate of circular dichroism enhancement, theoretical simulation results.

\section{AUTHOR INFORMATION}

\section{Corresponding Authors}

Jatish Kumar - Department of Chemistry, Indian Institute of Science Education and Research (IISER) Tirupati, Tirupati 517 507, India.

Email: jatish@iisertirupati.ac.in

F. Javier García de Abajo - ICFO-Institut de Ciencies Fotoniques, The Barcelona Institute of Science and Technology, 08860 Castelldefels (Barcelona), Spain; ICREA-Institució Catalana de Recerca i Estudis Avançats, Passeig Lluís Companys 23, 08010 Barcelona, Spain.

Email: javier.garciadeabajo@nanophotonics.es

Luciano Galantini - Dipartimento di Chimica, “Sapienza” Università di Roma, P. le A. Moro 5, 00185 Roma, Italy.

Email: luciano.galantini@uniroma1.it

\section{Authors}

Emilia Severoni - Dipartimento di Chimica, "Sapienza" Università di Roma, P. le A. Moro 5, 00185 Roma, Italy.

Sonia Maniappan - Department of Chemistry, Indian Institute of Science Education and Research (IISER) Tirupati, Tirupati 517 507, India.

Luis M. Liz-Marzán - CIC biomaGUNE, Basque Research and Technology Alliance (BRTA), Paseo de Miramón 182, 20014 Donostia-San Sebastián, Spain; Ikerbasque, Basque Foundation for Science, 48013 Bilbao, Spain; Centro de Investigación en Red de Bioingeniería, Biomateriales y Nanomedicina (CIBER-BBN), Paseo de Miramón 182, 20014 Donostia-San Sebastián, Spain.

\section{AUTHOR CONTRIBUTIONS}

LMLM, LG and JK conceived the project. LMLM coordinated the project. ES, SM and JK carried out the experiments. FJGA carried out the theoretical analysis and simulations. All authors 
analyzed the data and contributed to the manuscript preparation. All authors have given approval to the final version of the manuscript.

\section{NOTES}

The authors declare no competing financial interest.

\section{ACKNOWLEDGMENT}

Sapienza University of Rome is acknowledged for financial support (project ID RM116154F1B53DC8). J.K. acknowledges financial support from DST-SERB (Project No. CRG/2019/002715) and S.M. acknowledges DST-INSPIRE for fellowship. F.J.G.A. acknowledges support from ERC (Advanced Grant No. 789104-eNANO), the Spanish MINECO (MAT2017-88492-R, MAT86659-R, SEV2015-0522, and MDM-2017-0720), the Catalan CERCA Program, and Fundació Privada Cellex.

\section{REFERENCES}

(1) Liz-Marzán, L. M. Tailoring Surface Plasmons through the Morphology and Assembly of Metal Nanoparticles. Langmuir 2006, 22, 32-41.

(2) Caswell, K. K.; Wilson, J. N.; Bunz, U. H. F.; Murphy, C. J. Preferential End-to-End Assembly of Gold Nanorods by Biotin-Streptavidin Connectors. J. Am. Chem. Soc. 2003, 125, 13914-13915.

(3) Thomas, R.; Kumar, J.; George, J.; Shanthil, M.; Naidu, G. N.; Swathi, R. S.; Thomas, K. G. Coupling of Elementary Electronic Excitations: Drawing Parallels between Excitons and Plasmons. J. Phys. Chem. Lett. 2018, 9, 919-932.

(4) Huang, Y.; Ma, L.; Hou, M.; Li, J.; Xie, Z.; Zhang, Z. Hybridized Plasmon Modes and Near-Field Enhancement of Metallic Nanoparticle-Dimer on a Mirror. Sci. Rep. 2016, 6, 30011.

(5) Moskovits, M. Surface-Enhanced Raman Spectroscopy: A Brief Retrospective. J. Raman Spectrosc. 2005, 36, 485-496.

(6) Li, J. F.; Li, C. Y.; Aroca, R. F. Plasmon-Enhanced Fluorescence Spectroscopy. Chem. Soc. Rev. 2017, 46, 3962-3979.

(7) Kumar, J.; Liz-Marzán, L. M. Recent Advances in Chiral Plasmonics: Towards Biomedical 
Applications. Bull. Chem. Soc. Jpn. 2019, 92, 30-37.

(8) Sun, M.; Xu, L.; Banhg, J. H.; Kuang, H.; Alben, S.; Kotov, N. A.; Xu, C. Intracellular Localization of Nanoparticle Dimers by Chirality Reversal. Nat. Commun. 2017, 8, 1847.

(9) Hao, C.; Xu, L.; Sun, M.; Ma, W.; Kuang, H.; Xu, C. Chirality on Hierarchical SelfAssembly of Au@AuAg Yolk-Shell Nanorods into Core-Satellite Superstructures for Biosensing in Human Cells. Adv. Funct. Mater. 2018, 28, 1802372.

(10) González-Rubio, G.; Mosquera, J.; Kumar, V.; Pedrazo-Tardajos, A.; Llombart, P.; Solís, D. M.; Lobato, I.; Noya, E. G.; Guerrero-Martínez, A.; Taboada, J. M.; Obelleiro, F.; MacDowell, L. G.; Bals, S.; Liz-Marzán, L. M. Micelle-Directed Chiral Seeded Growth on Anisotropic Gold Nanocrystals. Science 2020, 368, 1472-1477.

(11) Lee, H. E.; Ahn, H. Y.; Mun, J.; Lee, Y. Y.; Kim, M.; Cho, N. H.; Chang, K.; Kim, W. S.; Rho, J.; Nam, K. T. Amino-Acid- and Peptide-Directed Synthesis of Chiral Plasmonic Gold Nanoparticles. Nature 2018, 556, 360-364.

(12) George, J.; George Thomas, K. Surface Plasmon Coupled Circular Dichroism of Au Nanoparticles on Peptide Nanotubes. J. Am. Chem. Soc. 2010, 132, 2502-2503.

(13) Kumar, J.; Eraña, H.; López-Martínez, E.; Claes, N.; Martín, V. F.; Solís, D. M.; Bals, S.; Cortajarena, A. L.; Castilla, J.; Liz-Marzán, L. M. Detection of Amyloid Fibrils in Parkinson’s Disease Using Plasmonic Chirality. Proc. Natl. Acad. Sci. U. S. A. 2018, 115, 3225-3230.

(14) Han, B.; Zhu, Z.; Li, Z.; Zhang, W.; Tang, Z. Conformation Modulated Optical Activity Enhancement in Chiral Cysteine and Au Nanorod Assemblies. J. Am. Chem. Soc. 2014, 136, 16104-16107.

(15) Zhou, C.; Duan, X.; Liu, N. DNA-Nanotechnology-Enabled Chiral Plasmonics: From Static to Dynamic. Acc. Chem. Res. 2017, 50, 2906-2914.

(16) Querejeta-Fernández, A.; Chauve, G.; Methot, M.; Bouchard, J.; Kumacheva, E. Chiral Plasmonic Films Formed by Gold Nanorods and Cellulose Nanocrystals. J. Am. Chem. Soc. 2014, 136, 4788-4793.

(17) Kneer, L. M.; Roller, E.-M.; V. Besteiro, L.; Schreiber, R.; O. Govorov, A.; Liedl, T. Circular Dichroism of Chiral Molecules in DNA-Assembled Plasmonic Hotspots. ACS Nano 2018, 12, 9110-9115.

(18) Layani, M. E.; Ben Moshe, A.; Varenik, M.; Regev, O.; Zhang, H.; Govorov, A. O.; 
Markovich, G. Chiroptical Activity in Silver Cholate Nanostructures Induced by the Formation of Nanoparticle Assemblies. J. Phys. Chem. C 2013, 117, 22240-22244.

(19) García-Etxarri, A.; Ugalgo, J. M.; Sáenz, J. J.; Mujica, V. Field-Mediated Chirality Information Transfer in Molecule-Nanoparticle Hybrids. J. Phys. Chem. C 2020, 124, 1560-1565.

(20) Madenci, D.; Egelhaaf, S. U. Self-Assembly in Aqueous Bile Salt Solutions. Curr. Opin. Colloid Interface Sci. 2010, 15, 109-115.

(21) Galantini, L.; di Gregorio, M. C.; Gubitosi, M.; Travaglini, L.; Vázquez Tato, J.; Jover, A.; Meijide, F.; Soto Tellini, V. H.; Pavel, N. V. Bile Salts and Derivatives: Rigid Unconventional Amphiphiles as Dispersants, Carriers and Superstructure Building Blocks. Curr. Opin. Colloid Interface Sci. 2015, 20, 170-182.

(22) di Gregorio, M. C.; Travaglini, L.; Del Giudice, A.; Cautela, J.; Pavel, N. V.; Galantini, L. Bile Salts: Natural Surfactants and Precursors of a Broad Family of Complex Amphiphiles. Langmuir 2019, 35, 6803-6821.

(23) Rahman, M. A.; Bam, M.; Luat, E.; Jui, M. S.; Ganewatta, M. S.; Shokfai, T.; Nagarkatti, M.; Decho, A. W.; Tang, C. Macromolecular-Clustered Facial Amphiphilic Antimicrobials. Nat. Commun. 2018, 9, 5231.

(24) Faustino, C.; Serafim, C.; Rijo, P.; Reis, C. P. Bile Acids and Bile Acid Derivatives: Use in Drug Delivery Systems and as Therapeutic Agents. Expert Opin. Drug Deliv. 2016, 13, 1133-1148.

(25) Cautela, J.; Severoni, E.; Redondo-Gómez, C.; di Gregorio, M. C.; Del Giudice, A.; Sennato, S.; Angelini, R.; D’Abramo, M.; Schillén, K.; Galantini, L. C-12 vs C-3 Substituted Bile Salts: An Example of the Effects of Substituent Position and Orientation on the Self-Assembly of Steroid Surfactant Isomers. Colloid Surf. B 2020, 185, 110556.

(26) Soto Tellini, V. H.; Jover, A.; Meijide, F.; Vázquez Tato, J.; Galantini, L.; Pavel, N. V. Supramolecular Structures Generated by a P-Tert-Butylphenylamide Derivative of Cholic Acid: From Vesicles to Molecular Tubes. Adv. Mater. 2007, 19 , 1752-1756.

(27) Manghisi, N.; Leggio, C.; Jover, A.; Meijide, F.; Pavel, N. V.; Soto Tellini, V. H.; Vázquez Tato, J.; Agostino, R. G.; Galantini, L. Catanionic Tubules with Tunable Charge. Angew. Chem., Int. Ed. 2010, 49, 6604-6607.

(28) Ali, M. R. K.; Snyder, B.; El-Sayed, M. A. Synthesis and Optical Properties of Small Au 
Nanorods Using a Seedless Growth Technique. Langmuir 2012, 28, 9807-9815.

(29) di Gregorio, M. C.; Ben Moshe, A.; Tirosh, E.; Galantini, L.; Markovich, G. Chiroptical Study of Plasmon-Molecule Interaction: The Case of Interaction of Glutathione with Silver Nanocubes. J. Phys. Chem. C 2015, 119, 17111-17116

(30) Zhu, Z.; Liu, W.; Li, Z.; Han, B.; Zhou, Y.; Gao, Y.; Tang, Z. Manipulation of Collective Optical Activity in One-Dimensional Plasmonic Assembly. ACS Nano 2012, 6, 2326-2332.

(31) Kaler, E. W.; Murthy, A. K.; Rodriguez, B. E.; Zasadzinski, J. A. N. Spontaneous Vesicle Formation in Aqueous Mixtures of Single-Tailed Surfactants. Science 1989, 245, 13711374.

(32) Jung, H. T.; Coldren, B.; Zasadzinski, J. A.; Iampietro, D. J.; Kaler, E. W. The Origins of Stability of Spontaneous Vesicles. Proc. Natl. Acad. Sci. U. S. A. 2001, 98, 1353-1357.

(33) Yu, W.; Yang, Y.; Chang, C. Cosolvent Effects on the Spontaneous Formation of Vesicles from 1 : 1 Anionic and Cationic Surfactant Mixtures. Langmuir 2005, 21, 6185-6193.

(34) Tomašić, V.; Štefanić, I.; Filipović-Vinceković, N. Adsorption, Association and Precipitation in Hexadecyltrimethylammonium Bromide/Sodium Dodecyl Sulfate Mixtures. Colloid Polym. Sci. 1999, 277, 153-163.

(35) O. Govorov, A.; Fan, Z.; Hernandez, P.; M. Slocik, J.; R. Naik, R. Theory of Circular Dichroism of Nanomaterials Comprising Chiral Molecules and Nanocrystals: Plasmon Enhancement, Dipole Interactions, and Dielectric Effects. Nano Lett. 2010, 10, 1374-1382.

(36) Zhang, H; Govorov A. O. Giant Circular Dichroism of a Molecule in a Region of Strong Plasmon Resonances between Two Neighboring Gold Nanocrystals. Phys. Rev. B 2013, 87, 075410.

(37) Josephson B. The Optical Properties of Some Bile Acids. Biochem. J. 1935, 29, 1484-1489

(38) Yu, R.; Liz-Marzán, L. M.; García de Abajo, F. J. Universal Analytical Modeling of Plasmonic Nanoparticles. Chem. Soc. Rev. 2017, 46, 6710-6724.

(39) García de Abajo, F. J.; Howie, A. Retarded Field Calculation of Electron Energy Loss in Inhomogeneous Dielectrics. Phys. Rev. B 2002, 65, 1154181.

(40) González-Rubio, G.; González-Izquierdo, J.; Bañares, L.; Tardajos, G.; Rivera, A.; Altantzis, T.; Bals, S.; Peña-Rodríguez, O.; Guerrero-Martínez, A.; Liz-Marzán, L. M. Femtosecond Laser-Controlled Tip-to-Tip Assembly and Welding of Gold Nanorods. Nano Lett. 2015, 15, 8282-8288. 
(41) Tato, J. V.; Tellini, V. H. S.; Trillo, J. V.; Alvarez, M.; Antelo, A.; Carrazana, J.; Jover, A.; Meijide, F. Nuevos Amidoderivados de Ácidos Biliares Funcionalizados en la Pposición 3 del Anillo A. Procedimientos para su Obtención y Aplicaciones. Spanish Patent P2005 01843, 2005.

(42) Anelli, P. L.; Lattuada, L.; Uggeri, F. One-Pot Mitsunobu- Staudinger Preparation of 3Aminocholan-24-oic Acid Esters from 3-Hydroxycholan-24-oic Acid Esters. Synth. Commun. 1998, 28, 109-117.

(43) Nikoobakht, B.; El-Sayed, M. A. Preparation and Growth Mechanism of Gold Nanorods (NRs) Using Seed-Mediated Growth Method. Chem. Mater. 2003, 15, 1957-1962.

(44) Schellman, J. A. Circular Dichroism and Optical Rotation. Chem. Rev. 1975, 75, 323-331.

(45) Jackson, J. D. Classical Electrodynamics 3rd Edition; John Wiley \& Sons, New York, 1999.

(46) van de Hulst, H. C. Light Scattering by Small Particles; Dover, New York, 1981.

(47) Yang, Z. J.; Antosiewicz, T. J.; Verre, R.; García de Abajo, F. J.; Apell, S. P.; Käll, M. Ultimate Limit of Light Extinction by Nanophotonic Structures. Nano Lett. 2015, 15, 76337638.

(48) Asenjo-Garcia, A.; García de Abajo, F. J. Dichroism in the Interaction between Vortex Electron Beams, Plasmons, and Molecules. Phys. Rev. Lett. 2014, 113, 066102. 


\section{TOC GRAPHIC}

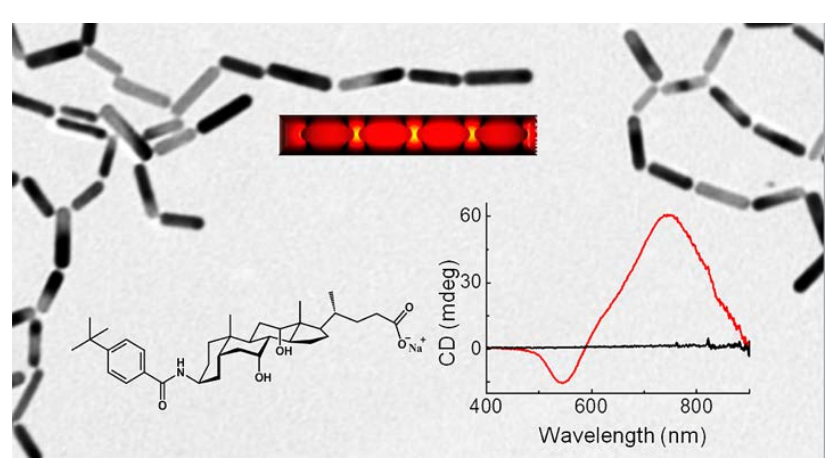

